\title{
Prevalencia y Distribución de Signos Degenerativos en Cóndilo de la ATM presentes en Radiografías Panorámicas en Población Chilena
}

\author{
Prevalence and Distribution of Degenerative Signs in ATM \\ Condyle in Panoramic Radiographs in a Chilean Population
}

Marcela Torrealba Triviño${ }^{1}$; Patricia Normandin Urzúa² ${ }^{2}$ Carmen Guzmán Zuluaga ${ }^{1}$ \& Gisaku Kuramochi Duhalde ${ }^{1}$

TORREALBA, T. M.; NORMANDIN, U. P.; GUZMÁN, Z. C. \& KURAMOCHI, D. G. Prevalencia y distribución de signos degenerativos en cóndilo de la ATM presentes en radiografías panorámicas en población chilena.Int. J. Morphol., 36(4):1519-1524, 2018.

RESUMEN: La articulación temporomandibular (ATM), como todas las articulaciones, es afectada por enfermedades inflamatorias o degenerativas, traumatismos, malformaciones, infecciones, quistes y tumores. La artrosis u osteoartrosis (OA) es la enfermedad degenerativa más frecuente de las articulaciones del cuerpo humano, incluyendo la ATM. El propósito de este estudio fue determinar la prevalencia de signos imagenológicos de trastornos degenerativos en el proceso condilar de la ATM, presentes en radiografías panorámicas digitales (RPD) de población chilena. Para ello se realizó un estudio de corte transversal que utilizó 651 radiografías de individuos adultos, en las que se buscó la presencia de los siguientes signos imagenológicos de osteoartrosis: esclerosis, pérdida de la cortical, erosión, osteofitos y quistes endocondrales. La prevalencia de signos radiográficos de OA fue de un $35 \%$ (n=230); donde el $50 \%$ de los individuos con signos de OA fueron mayores de 50 años, mientras la diferencia se distribuyó de forma homogénea en los distintos rangos etáreos. El signo más prevalente fue el osteofito (25\%) y la pérdida de cortical fue la menos observada (3\%). Además, la esclerosis presentó una prevalencia del $6 \%$, la erosión del $4 \%$ y los quistes endocondrales del $6 \%$. Por su parte la frecuencia de signos observada en hombres fue mayor, detectándose un aumento en relación directa con la edad independiente del sexo. La radiografía panorámica por su accesibilidad, baja dosis de radiación y constituir un examen de rutina, constituye un medio apropiado para pesquizar hallazgos de signos degenerativos de la ATM.

PALABRAS CLAVE: Articulación Témporomandibular (ATM); Prevalencia; Osteoartrosis; Población chilena.

\section{INTRODUCCIÓN}

Los trastornos o desórdenes temporomandibulares (TTM) afectan a la articulación temporomandibular (ATM), a los músculos de la masticación y las estructuras asociadas (Frugone et al., 2012). Estos se caracterizan por presentar una serie de signos y síntomas orofaciales de etiología multifactorial. La ATM es una de las articulaciones más complejas del cuerpo humano, esta complejidad puede explicar la aparición de alteraciones en ella a lo largo de la vida de un individuo, sin embargo, esto no significa que todos los individuos tengan que padecerlas (Mendiburu et al., 2016).

En general se observan distribuciones para sexo y edad muy diversas (Sandoval et al., 2015). Sin embargo, se podría estimar en forma conservadora que el porcentaje de individuos de la población general con algún TTM es del orden del 40-50 \% (Sandoval et al.).
La osteoartrosis (OA) es la enfermedad degenerativa más frecuente de las articulaciones del cuerpo humano, incluyendo la ATM (Cahlin \& Dahlström, 2011; Mendiburu et al.). Cuando no es tratada puede llegar a afectar significativamente la calidad de vida de los pacientes.

De los pacientes tratados por disfunción temporomandibular un $8 \%$ a $12 \%$ reciben el diagnóstico de osteoartrosis (OA). Estudios realizados mediante autopsias revelan que entre un $22 \%$ y un $40 \%$ de la población presentan OA (Martínez Blanco et al., 2004). Esto implica que alrededor de un 10-18 \% jamás es diagnosticado. El daño óseo degenerativo de la ATM y los cambios radiográficos que se pueden encontrar se representan como irregularidades del contorno óseo, pérdida de cortical ósea, disminución del volumen condilar, erosión, esclerosis y apla-

${ }^{1}$ Facultad de Odontología, Universidad Finis Terrae, Santiago, Chile

${ }^{2}$ Programa de Magíster en Imagenología, Facultad de Odontología, Universidad Finis Terrae, Santiago, Chile. 
namientos (tanto en el cóndilo como proceso condilar), formación de osteofitos (Quevedo et al., 2012; Santos-Tucto et al., 2014) que evidencian un sobrecrecimiento de hueso marginal y aparición de quistes subcondrales, como concavidades en el hueso (Solórzano Peláez et al., 1999; Quevedo et al.; Santos-Tucto et al.).

Los desórdenes de la ATM afectan a las personas fundamentalmente con la sensación de dolor, tanto crónico como agudo, y con la disfunción del Sistema Estomatognático, expresada en la limitación del movimiento mandibular, dificultad para comer y hablar que alteran significativamente la calidad de vida de los pacientes (Cömert Kiliç et al., 2015).

Es importante dimensionar la realidad local de los trastornos de la ATM para justificar el diseño de protocolos orientados a establecer un diagnóstico temprano, específicamente sobre los signos de trastornos degenerativos, que representan entre el $5 \%$ y $16 \%$ de los pacientes con TTMs (Cahlin \& Dahlström), mediante una observación acuciosa de la radiografía panorámica que los pacientes se toman de manera rutinaria. La detección precoz de estos trastornos permitiría el tratamiento apropiado por parte de los especialistas, lo cual, mejoraría la calidad de vida de quienes los padecen.

Por ello, el objetivo de este estudio fue conocer la prevalencia de signos imagenológicos asociados a trastornos degenerativos de la ATM, mediante el análisis de radiografías panorámicas digitales en población chilena.

\section{MATERIAL Y MÉTODO}

Se realizó un estudio de prevalencia, a partir de 651 radiografías panorámicas digitales (relación 1:1) anonimizadas de individuos adultos, mayores de 20 años entre abril del 2014 y septiembre del 2016 atendidos en el Servicio Radiológico de la Facultad de Odontología, Universidad Finis Terrae, Chile. Del total de participantes, el $67,3 \%(n=438)$ fueron mujeres y el 32,7 \% ( $n=213)$ hombres. Las RPD fueron tomadas con el equipo ORTHOPHOS XG 3D / Ceph (Sirona; Bensheim, Alemania). El presente estudio cuenta con la aprobación de su protocolo por parte del Comité Ético Científico (CEC) de la Universidad Finis Terrae (Resolución No 15/2017).

Se aplicaron los siguientes criterios de exclusión: Pacientes sometidos a cirugías de la ATM, con grandes deformaciones de forma y tamaño de la ATM, agenesia de cóndilo, anquilosis temporomandibular, imágenes con artefactos por movimiento e imágenes con distorsiones por mal posicionamiento del paciente.

La muestra fue obtenida mediante la técnica de muestreo aleatorio simple. De un universo de 1018 radiografías panorámicas, considerando un nivel de seguridad del $95 \%$, un $3 \%$ de precisión y tomando como referencia una prevalencia del $39 \%$ (Bakke et al., 2014), se estimó una muestra final de 651 radiografías que cumplieron con los criterios de elegibilidad (Fig. 1).
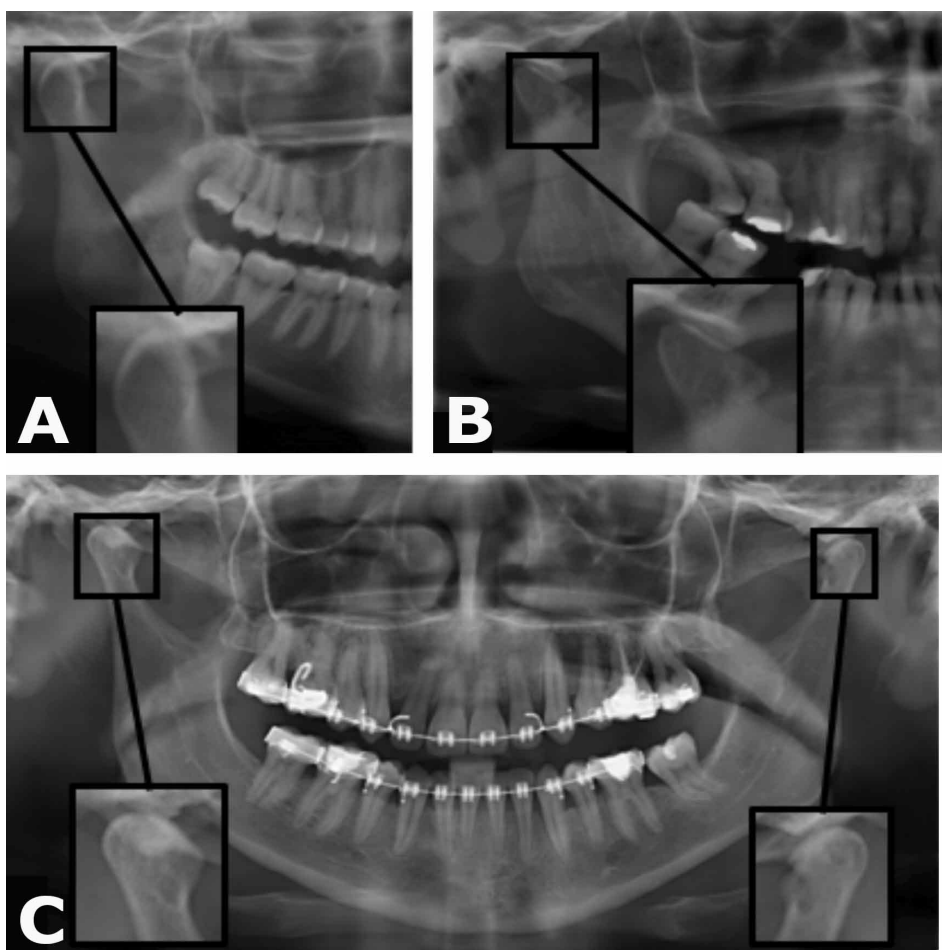

1520
Fig. 1. Radiografías panorámicas donde se pueden observar signos de OA. A: Rx. paciente sexo femenino, 20 años,presenta esclerosis en cóndilo derecho. B: Rx. paciente sexo femenino, 59 años, presenta erosión en cóndilo izquierdo C: Rx. paciente sexo masculino, 59, presenta aplanamiento, perdida de cortical, osteofitos y quistes endocondrales. D: Rx.paciente sexo femenino, 34 años, presenta osteofito en cóndilo derecho y erosión en cóndilo izquierdo. 
Tabla I. Distribución porcentual de OA según sexo y edad en la muestra.

\begin{tabular}{cccc}
\hline \multirow{2}{*}{ EDAD } & \multicolumn{2}{c}{ SEXO } & Total \\
\cline { 2 - 3 } & Femenino & Masculino & \\
\hline 20-29 años & 240 & 110 & 350 \\
30-39 años & 44 & 22 & 66 \\
40-49 años & 46 & 23 & 69 \\
50-59 años & 55 & 29 & 84 \\
>=60 años & 53 & 29 & 82 \\
TOTAL & 438 & 213 & 651 \\
\hline
\end{tabular}

Todas las radiografías panorámicas digitales se proyectaron en un monitor LED de 29 pulgadas (LG, model 29UM67) con una resolución 2560 x 1080 pixeles y fueron analizadas por un solo examinador. La confiabilidad intraobservador se evaluó realizando un doble análisis de un 10 $\%$ de las radiografías estudiadas, con una semana de diferencia entre la primera y segunda medición.

El rango de edad de los individuos osciló entre los 20 y 89 años. La muestra estuvo constituida por aproximadamente un $50 \%$ de personas entre 20 y 29 años mientras el porcentaje restante de los participantes se distribuyó de forma homogénea (10-12 \%) en cada uno de los otros grupos etáreos (Tabla I).

Las radiografías fueron clasificadas inicialmente según sexo, considerando cinco grupos etáreos diferentes (20$29,30-39,40-49,50-59$ y 60 y más años de edad). Tras lo cual, todas las imágenes radiográficas fueron estudiadas siguiendo el mismo protocolo de examinación: primero el pro- ceso condilar derecho del paciente y luego el izquierdo. Cada proceso condilar se analizó buscando la presencia de cinco signos radiográficos de osteoratrosis de la ATM: esclerosis ósea del hueso subcondral, pérdida de la cortical del cóndilo mandibular, erosión del cóndilo mandibular, presencia de osteofitos y de quistes endocondrales. Los resultados del examen fueron registrados en una planilla y para su análisis se utilizó el software Stata versión 14 (StataCorp; Texas; EEUU).

\section{RESULTADOS}

La prevalencia de signos de $\mathrm{OA}$ en el total de la muestra fue de un $35 \%(\mathrm{n}=230)$. Mientras que de acuerdo al sexo en mujeres fue del $34 \%(n=149)$ y en hombres del 38 $\%(\mathrm{n}=81)$.

La prevalencia de signos de $\mathrm{OA}$ de acuerdo al rango etáreo se muestra en la Figura 2 y su distribución de acuerdo a sexo y edad, en la Figura 3.

Considerando la frecuencia en el número de signos de osteoartrosis, del total de pacientes con signos de OA $(\mathrm{n}=230)$, un $78 \%(\mathrm{n}=179)$ presentaba sólo un signo, un 18 $\%(\mathrm{n}=42)$ dos y un $4 \%(\mathrm{n}=9)$ tres signos.

La frecuencia particular de cada signo obtenida fue la siguiente: del total de signos OA $(\mathrm{n}=291)$, la esclerosis correspondió a un $14 \%(\mathrm{n}=41)$, la pérdida de cortical correspondió a un $7 \%(\mathrm{n}=21)$, la erosión a un $9 \%(\mathrm{n}=27)$, los osteofitos a un $56 \%(\mathrm{n}=161)$ y los quistes endocondrales a un $14 \%(\mathrm{n}=41)$.

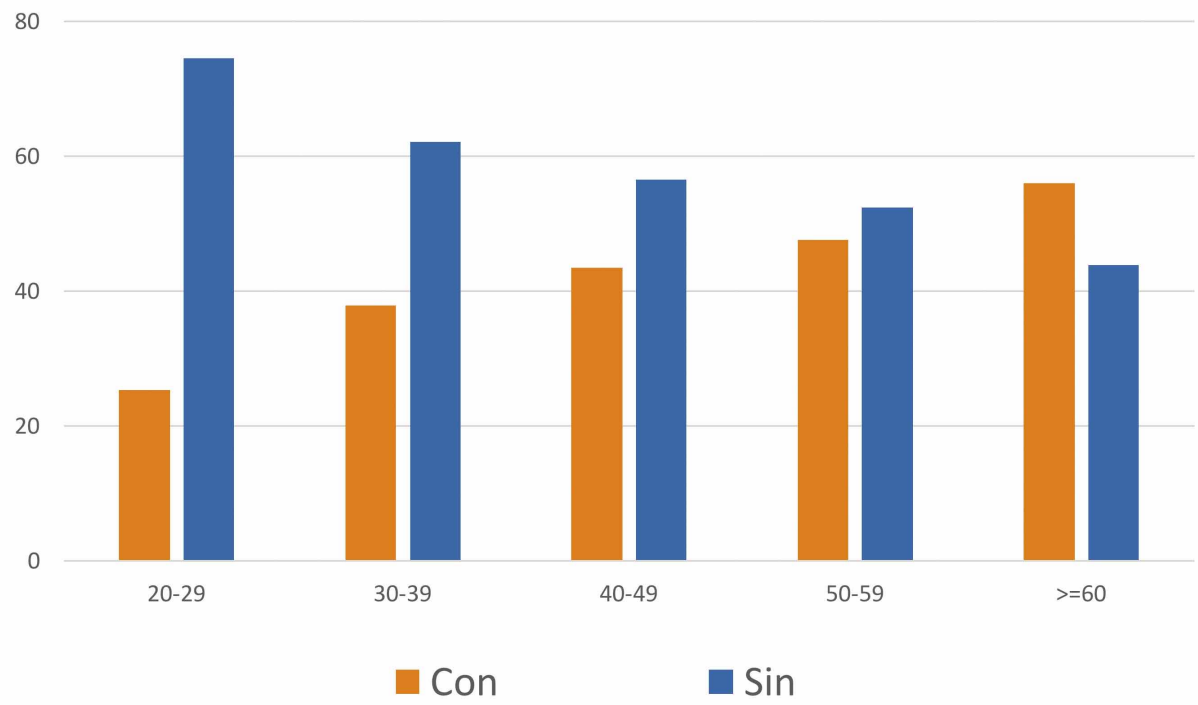

Fig. 2. Prevalencia de signos de OA de acuerdo al rango etáreo. 


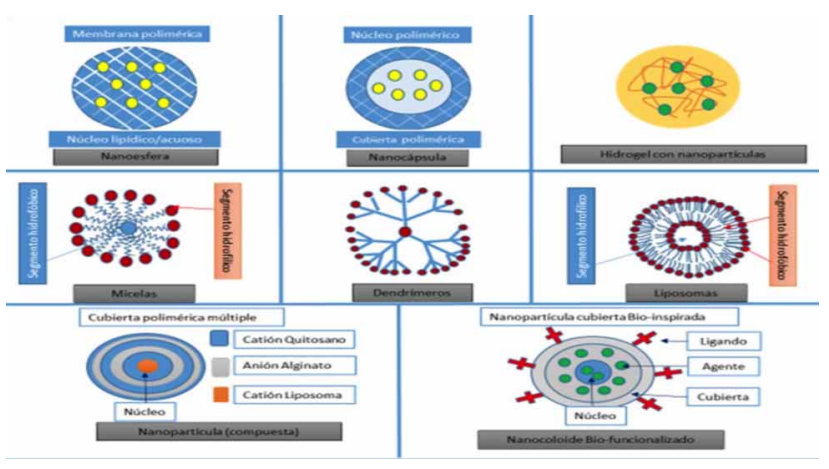

Fig. 3. Comparación de porcentajes de signos de osteoartrosis según sexo y edad.

La prevalencia en la muestra para cada uno de los signos de OA estudiados en este trabajo, se describe en la Tabla II. Mientras que las frecuencias particulares en relación al sexo se presentan en la Figura 4.

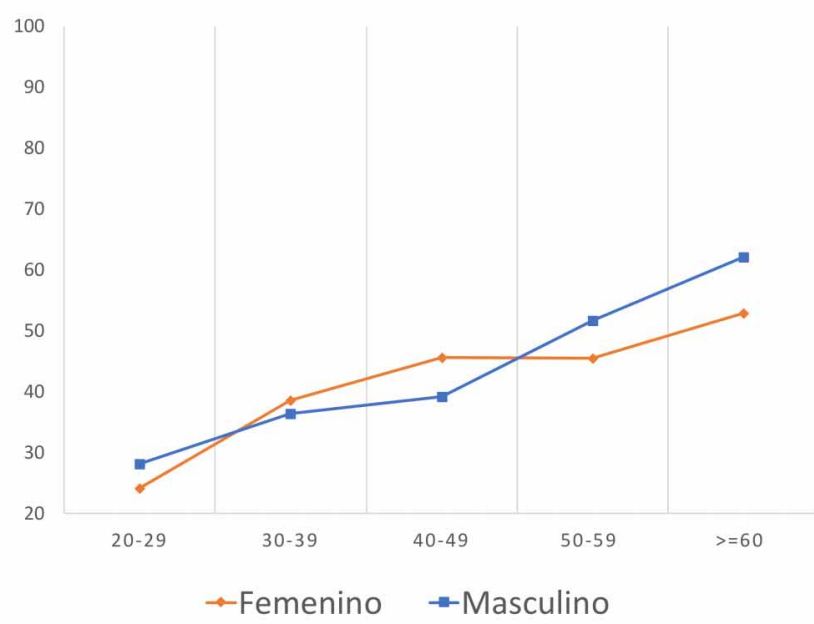

Fig. 4. Distribución de signos de osteoartrosis según sexo en porcentaje.

Tabla II. Prevalencia de los diferentes signos de osteoartrosis.

\begin{tabular}{lcc}
\hline \multicolumn{1}{c}{ S igno de OA } & $\mathrm{N}^{\circ}$ Casos & Prevalencia (\%) \\
\hline Esclerosis & 41 & 6 \\
Pérdida de cortical & 21 & 3 \\
Erosión & 26 & 4 \\
Osteofitos & 161 & 25 \\
Quistes endocondrales & 41 & 6
\end{tabular}

\section{DISCUSIÓN}

La identificación temprana de los TTMs es uno de los aspectos relevantes para el diagnóstico y la planificación terapéutica (Amaya González et al., 2015). Así el estu- dio radiológico en la $\mathrm{OA}$ es de gran importancia, pues permite pezquizar signos constantes aplicables a todas las articulaciones (Solórzano Peláez et al.).

La prevalencia de signos de OA obtenida en este estudio se enmarca dentro de los resultados reportados en la literatura, que oscilan entre un $18 \%$ y un 53,3\% (Broussard, 2005; Mendiburu et al.). Comparativamente en los trabajos realizados con CBTC los niveles de prevalencia observados son mayores (Bakke et al.); esta diferencia se explicaría por limitaciones propias de las RPD, como son su bidimensionalidad y la sobreproyección de estructuras maxilofaciales (Hunter \& Kalathingal, 2013); condicionantes que dificultan una identificación temprana de las lesiones. En contraposición, el CBTC entrega una información más acabada de la articulación (Larheim et al., 2015), ya que permite detectar cambios morfológicos sutiles asociados a OA (Tanaka et al., 2008) producto de una evaluación en los tres planos del espacio y sin sobreproyección (Broussard; Barghan et al., 2012; Larheim et al.).

En general, la contrastación de los valores de prevalencia disponibles con los encontrados en este estudio, ha sido una tarea compleja, debido a la diversidad de criterios utilizados en la inclusión de signos asociados a OA. En lo particular, hemos incluido la esclerosis como un signo de OA, aun cuando en otros estudios sólo se considera como un cambio adaptativo si no es generalizada (Hunter \& Kalathingal; Rojas-Martínez \& Lozano-Castro, 2014) y excluido el aplanamiento condilar ya que en la actualidad se ha descrito que ésta puede ser una mera desviación de forma o resultado de un proceso adaptativo (Schultz et al., 2011; Hunter \& Kalathingal; Rojas-Martínez \& Lozano-Castro).

Con respecto al sexo, las prevalencias encontradas difieren con lo expuesto en la literatura científica, donde los trastornos degenerativos de la ATM tienen una representación mayor en mujeres (dos Anjos Pontual et al., 2012; Sandoval et al.; Bustillos et al., 2016). Esta discrepancia podría explicarse señalando que tales estudios fueron realizados en pacientes con síntomas o signos de TTM, o bien porque las mujeres además de expresar en forma más acentuada la sintomatología que los hombres, tienden a consultar con mayor frecuencia, frente a los signos y/o síntomas que perciben (Pérez Romero et al., 2011).

En cuanto a la distribución de los signos de $\mathrm{OA}$ en los distintos grupos etarios, los valores encontrados en este estudio revelan una relación directamente proporcional con la edad y una distribución similar a la encontrada en la evidencia científica. (dos Anjos Pontual et al.; Hunter \& Kalathingal.). Al respecto, que la presencia de la enfermedad sea mayor conforme aumenta la edad, resulta esperable dado que se trata de 
una enfermedad degenerativa (Wiese et al., 2008; dos Anjos Pontual et al.; Hunter \& Kalathingal; Rojas-Martínez \& Lozano-Castro).

El análisis individual de los diferentes signos de OA, entrega para la esclerosis una prevalencia del $6 \%$, valor muy inferior a los reportados por Santos-Tucto et al. de 29,9\% y $31,8 \%$ entre una articulación y otra. Esto se podría deber a que en este último se utilizó CBTC, modalidad de examen capaz de discriminar cambios muy leves, a diferencia de la radiografía panorámica (Barghan et al.). No obstante, un estudio previo (Lopes et al., 2005) realizado tambien mediante CBTC, la prevalencia informada fue bastante similar a la de este trabajo, con un 6,7\%. Estas diferencias y similitudes con la literatura inducen a pensar que la esclerosis podría ser un signo variable y que su presencia estaría condicionada por otros factores no evaluados en este protocolo.

En relación a la pérdida de hueso cortical, la prevalencia obtenida $(3 \%)$ se distribuyó de manera heterogénea en los distintos grupos etarios, aunque tanto hombres como mujeres fueron afectados de manera similar. Este valor de prevalencia dista del 25,5\% encontrado en una investigación realizada con CBTC (Nah, 2012), discrepancia atribuible a la elevada sensibilidad de la técnica para detectar pérdidas de tejido óseo (López López et al., 2005) y a diferencias en la composición de las muestras sujetas a estudio.

La erosión por su parte, se distribuyó de manera homogénea entre todos los grupos, sin diferencias entre sexos y con una prevalencia menor al $8 \%$ informado en otro estudio realizado en RPD (Quevedo et al.), pero bastante inferior a la obtenida en CBTC (Reyes Velázquez \& Pérez Romero, 2011) que varió de un $20,1 \%$ a 22,1 \% para las ATMs derecha e izquierda, respectivamente. La disimilitud observada para este signo, estaría determinada por los criterios diagnósticos utilizados en cada estudio, las variaciones inter-observador y la sensibilidad de la técnica radiológica utilizada.

Otro de los signos de OA, los osteofitos, presentaron la prevalencia más alta y una presencia marcada en hombres, en todos los grupos etarios. Este valor resulta mayor que lo informado en otros estudios (3-9 \%) (Broussard; Reyes Velázquez \& Pérez Romero), pero muy inferior a los resultados obtenidos por Lopes et al. realizado con CBTC (57\%), lo cual probablemente además de lo atribuible a la técnica radiográfica, tambien respondería a una condición propia de la muestra estudiada.

La prevalencia de quistes endocondrales fue de un 6 $\%$, no observándose una distribución diferenciada en relación al sexo. En estudios previos se reportó una prevalencia del $3,4 \%$ (Cömert Kiliç et al.) y valores que variaron entre 1,9 y
0,6 \% entre la ATM derecha y ATM izquierda (Santos-Tucto et al.). Por lo tanto, se podría asumir que la formación de quistes es también un signo variable de la OA cuya presencia podría estar influenciada por diferentes factores y condiciones individuales.

La radiografía panorámica resulta un método útil en la detección de signos de OA, como se comprueba al comparar nuestros resultados con los reportados en la literatura respecto de otras técnicas imagenológicas (Hintze et al., 2009). Además de constituir un examen de rutina ampliamente utilizado debido a su disponibildad, costo y menor exposición, lo que supone una ventaja (Fuentes et al., 2015, 2017). No obstante, es fundamental considerar también sus desventajas, como la sobreproyección, visión bidimensional de una estructura tridimensional y su baja capacidad de discriminar cambios estructurales en estadios iniciales (Hunter $\&$ Kalathingal). Condicionantes que representaron para nuestro análisis una limitación en la detección de los distintos signos de OA.

En consecuencia la radiografía panorámica constituye un recurso inicial para estudiar cambios morfológicos que afecten a la ATM pero que precisa del uso de técnicas como CBTC para la identificación temprana de signos degenerativos.

Por su parte, la falta de consenso respecto de los signos asociados a OA obliga a recomendar el desarrollo de un número mayor de estudios para dilucidar aquellos aspectos que permitan una mejor comparabilidad entre los mismos.

Los resultados obtenidos en el presente trabajo nos permiten concluir que la prevalencia de OA en la población estudiada es de un $35 \%$ con una representación mayor en hombres y cuya frecuencia se incrementa conforme aumenta la edad. Tanto el valor de la prevalencia de OA como la correspondiente a cada signo se ven afectados por la modalidad de análisis radiográfico y consideraciones metodológicas.

TORREALBA, T. M.; NORMANDIN, U. P.; GUZMÁN, Z. C. \& KURAMOCHI, D. G. Prevalence and distribution of degenerative Signs in ATM Condyle in panoramic radiographs in a Chilean population. Int. J. Morphol., 36(4):1519-1524, 2018.

SUMMARY: The temporomandibular joint (TMJ), like all joints, is affected by inflammatory or degenerative diseases, traumatisms, malformations, infections, cysts and tumors. Osteoarthrosis (OA) is the most common degenerative disease of the joints of the human body, including the temporomandibular joint. The purpose of this study was to estimate the prevalence of imaging signs of degenerative disorders in the TMJ condyle, present in digital panoramic radiographs in Chilean population. A cross-sectional study was conducted using 651 radiographs of adult individuals. The presence of the following imaging signs of osteoarthrosis was recorded: sclerosis, 
cortical loss, erosion, osteophytes and endochondral cysts. The prevalence of radiographic signs of OA was $35 \%(n=230)$; where 50 $\%$ of the individuals with signs of OA were older than 50 years, while the difference was distributed homogeneously in the different age ranges. The most prevalent sign was osteophyte $(25 \%)$ and cortical loss was the least observed (3\%). In addition, sclerosis presented a prevalence of $6 \%$, erosion of $4 \%$ and endochondral cysts of $6 \%$. On the other hand, the frequency of signs observed in men was greater, detecting an increase in direct relation with age independent of sex. The data of this study allows us to conclude that the prevalence of OA in the studied population was $35 \%$ with a higher representation in men and older people. Both the value of the prevalence of OA and that corresponding to each sign are affected by the modality of radiographic analysis and methodological considerations.

KEY WORDS: Temporomandibular Joint (TMJ); Prevalence; Osteoarthrosis; Osteoarthritis; Chilean Population.

\section{REFERENCIAS BIBLIOGRÁFICAS}

Amaya González, S. Y.; Casanova Antolínez, C. M.; Barrera Chaparro, J. P.; Benavides Bonilla, R. A.; Sánchez Chaparro, N. Y. \& Buriticá Guarnizo, A. Prevalencia de trastornos de la articulación temporomandibular según los criterios diagnósticos para la investigación en pacientes preortodónticos. Univ. Odontol., 33(71):19-28, 2015.

Bakke, M.; Petersson, A.; Wiesel, M.; Svanholt, P. \& Sonnesen, L. Bony deviations revealed by cone beam computed tomography of the temporomandibular joint in subjects without ongoing pain. J. Oral Facial Pain Headache, 28(4):331-7, 2014.

Barghan, S.; Tetradis, S. \& Mallya, S. Application of cone beam computed tomography for assessment of the temporomandibular joints. Aust. Dent. J., 57 Suppl. 1:109-18, 2012.

Broussard, J. S. Jr. Derangement, osteoarthritis, and rheumatoid arthritis of the temporomandibular joint: implications, diagnosis, and management. Dent. Clin. North Am., 49(2):327-42, 2005

Bustillos, L.; Manoocheri, A.; Serrano, M. \& Zabala, S. Alteraciones temporomandibulares. Revisión de la literatura. Acta Bioclin., 6(12):10721, 2016.

Cahlin, B. J. \& Dahlström, L. No effect of glucosamine sulfate on osteoarthritis in the temporomandibular joints--a randomized, controlled, short-term study. Oral Surg. Oral Med. Oral Pathol. Oral Radiol. Endod., 112(6):760-6, 2011.

Cömert Kiliç, S.; Kiliç, N. \& Sümbüllü, M. A. Temporomandibular joint osteoarthritis: cone beam computed tomography findings, clinical features, and correlations. Int. J. Oral Maxillofac. Surg., 44(10):1268-74, 2015.

dos Anjos Pontual, M. L.; Freire, J. S.; Barbosa, J. M.; Frazão, M. A. \& dos Anjos Pontual, A. Evaluation of bone changes in the temporomandibular joint using cone beam CT. Dentomaxillofac. Radiol., 41(1):24-9, 2012.

Frugone, R.; Oliva, P.; Jimenez, A. \& Zambrano, G. Revisión bibliográfica manejo de trastornos temporomandibulares. Revisión entre 2007 y 2011. Rev. Dent. Chile, 103(1):16-23, 2012.

Fuentes, R.; Arias, A.; Bucchi, C.; Saravia, D. \& Dias, F. Prevalence and morphometric characteristics of the mandibular incisive canal through panoramic radiographs in a Chilean population. Int. J. Morphol., 35(3):9317, 2017.

Fuentes, R.; Cantín, M.; Ottone, N. E. \& Bucchi, C. Characterization of bone components of the temporomandibular joint. A literature review. Int. J. Morphol., 33(4):1569-76, 2015.

Hintze, H.; Wiese, M. \& Wenzel, A. Comparison of three radiographic methods for detection of morphological temporomandibular joint changes: panoramic, scanographic and tomographic examination. Dentomaxillofac Radiol., 38(3):134-40, 2009.

Hunter, A. \& Kalathingal, S. Diagnostic imaging for temporomandibular disorders and orofacial pain. Dent. Clin. North Am., 57(3):405-18, 2013.
Larheim, T. A.; Abrahamsson, A. K.; Kristensen, M. \& Arvidsson, L. Z. Temporomandibular joint diagnostics using CBCT. Dentomaxillofac. Radiol., 44(1):20140235, 2015

López López, J.; Chimenos Küstner, E.; Blanco Carrión, A.; Reselló Llabrés, X. \& Jané Salas, E. Diagnóstico por la imagen de los trastornos de la articulación craneomandibular. Av. Odontoestomatol., 21(2):71-88, 2005.

Martínez Blanco, M.; Bagán, J. V.; Fons, A. \& Poveda Roda, R. Osteoartrosis de la articulación temporomandibular: Estudio clínico y radiológico de 16 pacientes. Med. Oral Patol. Oral Cir. Bucal, 9(2):106-15, 2004.

Mendiburu, Z. C. E.; Peñaloza, C. R.; Carrillo, M. J.; Cárdenas, E. R. A.; Cortés, C. D. \& Cervera, G. J. E. Signos y síntomas en la articulación temporomandibular de sujetos geriátricos con artritis, antes-después de fisioterapia. Odovtos Int. J. Dent. Sci., 18(2):51-9, 2016.

$\mathrm{Nah}, \mathrm{K}$. S. Condylar bony changes in patients with temporomandibular disorders: a CBCT study. Imaging Sci. Dent., 42(4):249-53, 2012.

Pérez Romero, G. A. \& Reyes Velázquez, J. O. Patología de la articulación temporomandibular y su tratamiento. Med. Oral, 13(3):78-83, 2011.

Quevedo, P. M.; Machado, M. E. \& Fasanella, M. Evaluación de la morfología mandibular en pacientes con disfunción Temporo Mandibular a través de la radiografía panorámica. Oral, 13(41):845-8, 2012.

Reyes Velázquez, J. O. \& Pérez Romero, G. A. Etiología y diagnóstico de las enfermedades de las articulaciones temporomandibulares. Med. Oral, 13(3):75-7, 2011

Rojas-Martínez, C. \& Lozano-Castro, F. E. Diagnóstico clínico y aspecto psicosocial de trastornos temporomandibulares según el índice CDI/TTM en estudiantes de odontología. Rev. Estomatol. Hered., 24(4):229-38, 2014.

Sandoval, I.; Ibarra, N.; Flores, G.; Marinkovic, K.; Díaz, W. \& Romo, F. Prevalence of temporomandibular disorders according to RDC/TMD in older people of Santiago, Chile. Int. J. Odontostomat., 9(1):73-8, 2015.

Santos-Tucto, T. \& Gonzales-Olaza, H. Cambios osteoartrósicos condíleos relacionados al espesor de la superficie articular del temporal y espacio articular temporomandibular según Tomografía Computarizada Cone Beam. KIRU, 11(1):56-68, 2014

Schultz, R.; Moya, M.; Reuss, C.; Ivanovic, M. \& Díaz, M. Relación entre los diagnosticos de eje I (físico) y eje II (psicosocial) según el criterio de diagnóstico para la investigación de los trastornostemporomandibulares (RDI/ TTM) en una población chilena. Rev. Dent. Chile, 102(3):24-9, 2011.

Solórzano Peláez, A. L.; González Blanco, O.; Balda Zavarce, R. \& GarcíaArocha, C. Osteoartritis de la articulación temporomandibular. Parte III. Acta Odontol. Venez., 37(3), 1999. Disponible en: https:// www.actaodontologica.com/ediciones/1999/3/ osteoartritis_articulacion_temporomandibular_manifestaciones_histopatologicas.asp

Tanaka, E.; Detamore, M. S. \& Mercuri, L. G. Degenerative disorders of the temporomandibular joint: etiology, diagnosis, and treatment. J. Dent. Res., 87(4):296-307, 2008

Wiese, M.; Svensson, P.; Bakke, M.; List, T.; Hintze, H.; Petersson, A.; Knutsson, K. \& Wenzel, A. Association between temporomandibular joint symptoms, signs, and clinical diagnosis using the RDC/TMD and radiographic findings in temporomandibular joint tomograms. J. Orofac. Pain, 22(3):239-51, 2008.

\author{
Dirección para correspondencia: \\ Dr. Gisaku Kuramochi Duhalde \\ Departamento de Odontología Restauradora \\ Facultad de Odontología \\ Universidad Finis Terrae \\ Av. Pedro de Valdivia 1509 \\ Santiago \\ CHILE
}

Email: gkuramochi@gmail.com

Recibido: 22-03-2018

Aceptado:12-09-2018 